\title{
Documentos
}

\section{CALIDAD DE VIDA EN MUJ ERES CON CÁNCER CÉRVICO UTERINO}

\author{
Luz María Montes L.a , María José Mullins P.a, María Teresa Urrutia S.1,a \\ ${ }^{1}$ Departamento Salud de la Mujer. Escuela de Enfermería. Pontificia Universidad Católica de Chile.
}

a Enfermera-Matrona.

\section{RESUMEN}

Antecedentes: El cáncer cérvico uterino $(\mathrm{CaCu})$ es la cuarta causa de muerte por cáncer en Chile y la primera entre los 20 a 44 años. Debido a efectivos métodos de pesquisa y tratamientos oportunos, cada vez es mayor el número de usuarias que viven libres de enfermedad, lo que hace fundamental valorar la calidad de vida (CV). Objetivo: Revisar la literatura con el objetivo de conocer de qué manera se afecta la CV de estas mujeres. Resultados: La revisión de literatura muestra una gran cantidad de instrumentos utilizados para medir $\mathrm{CV}$ en mujeres con $\mathrm{CaCu}$, principalmente genéricos, como también cuestionarios que evalúan aspectos por separado. La dimensión más afectada durante todo el proceso de enfermedad, es la dimensión sexual. La modalidad de tratamiento se considera clave en la repercusión en esta dimensión; la radioterapia al ser un tratamiento localizado produce alteraciones físicas que repercuten en la sexualidad, en cambio la cirugía afecta mayormente el aspecto psicológico. Conclusión: La CV en las mujeres con $\mathrm{CaCu}$ se ve alterada en todas sus dimensiones, principalmente en la dimensión sexual. Se postula la importancia de considerar la CV como un parámetro útil de evaluación, para de esta manera brindar una intervención oportuna, centrada en la necesidad que ellas presentan.

\section{PALABRAS CLAVES: Calidad de vida, cáncer cérvico uterino, sexualidad}

\section{SUMMARY}

Background: Cervical cancer is the fourth most frequent cause of death among women in Chile, and the leading cause of death among women between the ages of 20 and 44 . Effective screening methods and opportune treatment has resulted in a growing number of women whose illness is in remission, making an evaluation of the quality of life of these women over the stages of the illness worthwhile. Objective: This article reviews how different aspects of the patient's quality of life (QOL) are affected by cervical cancer. Results: The literature contains many measures of QOL, most of which are generic QOL instruments, as well as a wide range of questionnaires used to evaluate specific aspects of QOL. The sexual dimension of QOL suffers the most marked deterioration for women with cervical cancer. The treatment method employed determines the effect on sexual QOL: while radiotherapy produces local physical alterations which directly affect sexual QOL, surgery principally affects the psychological aspects of sexuality. Conclusion: This review concludes that QOL in women with cervical cancer deteriorates over all its dimensions, most especially sexual QOL. This makes it important to consider QOL when evaluating these patients. Opportune intervention is suggested, especially in those aspects that deteriorate most.

KEY WORDS: Quality of life, cervical cancer, sexuality 


\section{INTRODUCCIÓN}

El Cáncer cérvico uterino $(\mathrm{CaCu})$ es una de las principales causas de muerte por cáncer en la mujer en países subdesarrollados. A pesar de tener demostrado un gran potencial de prevención secundaria, continua presentando tasas elevadas de mortalidad. En nuestro país se ha constituido en la primera causa de muerte en el grupo entre 20 a 44 años y la sexta en el grupo de 45 a 64 años. A pesar que las cifras de $\mathrm{CaCu}$ han ido en disminución, sigue siendo un problema importante de salud en Chile, dado su alto impacto social y económico, y además porque afecta principalmente a mujeres cada vez más jóvenes y en edad reproductiva $(1,2)$.

El CaCu en Chile es una de las prioridades en salud pública, siendo una de las 25 patologías incluidas en la Reforma de Salud desde julio de 2005. Cabe destacar que en ese programa uno de los objetivos sanitarios es mejorar la calidad de vida (CV) de las mujeres afectadas (3).

La presente revisión tiene como objetivo conocer de qué manera las diversas dimensiones de la $\mathrm{CV}$ se ven afectadas en mujeres con $\mathrm{CaCu}$, con el propósito de aportar al conocimiento de los profesionales de la salud y fomentar la valoración y el cuidado integral de ellas.

\section{ANÁLISIS DEL TEMA}

Actualmente los temas relacionados con CV son reconocidos como factores determinantes en el costo beneficio del tratamiento en pacientes con cáncer $(4,5)$. La tasa de pacientes con $\mathrm{CaCu}$ libres de enfermedad ha ido en aumento dada la detección temprana y la efectividad y disponibilidad de los tratamientos actuales. Esto significa un aumento en la cantidad de años de vida, pero muchas veces un deterioro en su calidad impactando las diversas dimensiones de la CV. Por este motivo se hace indispensable valorar la CV de las mujeres afectadas y el impacto del cáncer y su tratamiento $(2,6-10)$.

No existe una definición única para $\mathrm{CV}$, sin embargo, existe un consenso en cuanto a su carácter multidimensional y subjetivo $(11,12)$. La calidad de vida es una noción humana subjetiva que se relaciona con el grado de satisfacción que tiene la persona con su situación física, estado emocional, vida familiar, amorosa, y social, así como con el sentido que le atribuye a su vida (11). La OMS (13) la define como "la percepción del individuo de su posición en la vida en el contexto de la cultura y sistemas de valores en los que vive y en relación con sus objetivos, expectativas, estándares y preocupaciones".

La CV se ha transformado en un índice innovador en la atención de salud. Los índices clásicos han sido la mortalidad, morbilidad, expectativa de vida, entre otros, sin embargo éstos no son suficientes al momento de evaluar el bienestar de las usuarias de manera integral. Esta situación ha hecho necesario desarrollar mediciones de CV que permitan entregar un cuidado global, ya que incluyen no tan solo el aspecto físico. Al respecto cabe señalar que mujeres en período de remisión de $\mathrm{CaCu}$ presentan niveles aceptables de salud global, sin embargo, persisten con molestias en otras áreas, lo que estaría reflejando un ajustamiento a la vida posterior al cáncer $(9,10)$.

Para objetivar las mediciones de CV, se han elaborado distintos instrumentos, y en el caso específico del $\mathrm{CaCu}$, estos son variados, caracterizándose principalmente por ser instrumentos genéricos (6), a los que se les agregan instrumentos de valoración específicos para usuarias con cáncer.

El aspecto físico es la dimensión más evaluada por el equipo de salud, pero quizás una de las menos afectadas en estas mujeres. Esta situación se ve reflejada en la publicación realizada por De Groot y cols (14) donde constatan que las preocupaciones psicológicas en mujeres con $\mathrm{CaCu}$ pueden persistir durante los dos primeros años posteriores al tratamiento; en cambio las preocupaciones por los síntomas físicos no sobrepasan los tres meses post tratamiento.

\section{Dimensión sexual}

Al ser el $\mathrm{CaCu}$ un cáncer ginecológico resulta evidente que la dimensión sexual pueda ser la más afectada; esto se sustenta en la gran cantidad de literatura que existe al respecto. Esta dimensión se afecta fundamentalmente por el tipo de tratamiento utilizado (15). La modalidad de tratamiento que mayor afecta el área afectiva y psicológica de la sexualidad es la cirugía de Wertheim Meiggs, seguido por la exanteración pélvica y luego por la radioterapia. Las mujeres que reciben radioterapia combinada (externa y braquiterapia) obtienen mayores dificultades en el aspecto físico de la sexualidad que las que reciben radioterapia simple. La quimioterapia es la modalidad de tratamiento que tiene una menor repercusión en el ámbito sexual $(5,8)$.

Las mujeres que reciben tratamiento combinado (cirugía y radioterapia) reinician más tardía- 
mente la actividad sexual que las que fueron sometidas sólo a cirugía (5). Según un estudio realizado por Hawighorst-Knapstein y cols (8), el realizar cirugía reconstructiva a mujeres sometidas a exanteración pélvica favorece su vida sexual. Las mujeres que no son sometidas a cirugías reconstructivas, reportan un deterioro en el funcionamiento sexual al presentar mayores impedimentos físicos y menor autoestima.

La radioterapia, se describe como la modalidad de tratamiento que más altera el aspecto físico de la sexualidad, produciendo disfunción sexual persistente durante los dos años posteriores. En un estudio realizado por Jensen y cols (16), se señala que las alteraciones sexuales referidas por pacientes sometidas a radioterapia en los primeros dos años posteriores al tratamiento, incluyeron: ausencia o falta de libido $(85 \%)$, falta de lubricación vaginal $(35 \%)$, dispareunia $(55 \%)$, disconformidad con la vida sexual $(30 \%)$ y pareja con libido disminuido (40\%). La estrechez vaginal dificulta la penetración por lo que un $30 \%$ de las pacientes tienen gran dificultad de completar el coito, mientras que para un $45 \%$ éste fue imposible.

El reinicio de la actividad sexual tras haber recibido el tratamiento, es recordado como un evento psicológicamente traumático. Un número de mujeres expresan que el miedo era tan grande en sus primeras experiencias sexuales post tratamiento, que se describen como "emocionalmente desconectadas" (5). Las mujeres refieren temor e inseguridad de no poder satisfacer sexualmente a sus parejas, y por consecuencia sienten que ellos buscarán satisfacción sexual en otro lado; refieren que el indicador de satisfacción sexual en los hombres es tener coito, por lo tanto muchas de ellas desafían sus dificultades físicas para satisfacer a sus parejas. Esto se observa más frecuentemente en parejas con una mala comunicación, situación que les impide hablar de forma directa y abierta sobre estas dificultades (5). La mala comunicación también repercute en las parejas de las mujeres con $\mathrm{CaCu}$, quienes señalan estar asustados de reiniciar la actividad sexual por miedo a causarles dolor o daño físico; las mujeres en cambio interpretan este comportamiento como desinterés o rechazo hacia ellas como mujer. Esta situación es más pronunciada en el caso de mujeres con radioterapia (5).

Al respecto, la comunicación del profesional con la usuaria es un factor importante a considerar, para ayudarles a enfrentar y superar las secuelas sexuales del tratamiento (5). Debiera existir continuidad en esta comunicación durante todo el proceso de enfermedad.

\section{Dimensión psicológica}

El CaCu es el cáncer que reporta los mayores índices de depresión (17), y en comparación con otros cánceres, como el de mama y de endometrio, es el que reporta las mayores aflicciones emocionales. Esto se debe a que el diagnóstico temprano y la efectividad del tratamiento contribuyen a que estén en promedio más años libres de enfermedad, lo que hace que vivan más tiempo con la amenaza de la recidiva $(14,18)$.

La aceptación inicial del diagnóstico de cáncer, su tratamiento y los posibles efectos secundarios son un componente importante para las habilidades de afrontamiento. Una actitud realista, determinante y una participación activa en el proceso de tratamiento permite, a algunas pacientes, enfrentar el diagnóstico, los procedimientos desagradables y los efectos secundarios con menor estrés (5).

Existen múltiples preocupaciones en las mujeres con $\mathrm{CaCu}$, entre las que predominan el apoyo social, el estrés general, el bienestar familiar, la vida sexual y de pareja y los síntomas físicos asociados al tratamiento (17). Un sentimiento recurrente es la preocupación constante por su enfermedad y el miedo a la recidiva. En un estudio realizado por Wenzel y cols (10), un $39 \%$ de las pacientes atribuye su preocupación actual al cáncer, $27 \%$ expresan que cosas externas hacen pensar en su cáncer y un $26 \%$ tratan de evitar desanimarse cuando piensan en el diagnóstico. Klee y cols (9), señalan que las pacientes refirieron depresión y preocupación al término del tratamiento y a los tres meses siguientes; esta situación bajó a niveles normales posterior a los seis meses. Sin embargo, tras dos años post tratamiento las mujeres aun refieren preocupación por su enfermedad.

Uno de los temas que preocupa a las mujeres es la infertilidad, dado el gran impacto en la feminidad y en el rol reproductivo. Las mujeres experimentan sentimientos de pérdida, los que influyen negativamente en su relación de pareja y dificultan la posibilidad de poder formar, de manera satisfactoria, una posterior relación $(5,10,19,20)$. Esta situación se ve reflejada en el estudio realizado por Wenzel y cols (10), en donde señalan mayores preocupaciones reproductivas en las usuarias con $\mathrm{CaCu}$ que en las mujeres con ausencia de enfermedad: tristeza de no poder tener hijos $(31 \%)$, incapacidad de hablar abiertamente sobre 
fertilidad $(30 \%)$, frustración relacionada a infertilidad $(25 \%)$ y luto por la pérdida de la capacidad reproductiva $(25 \%)$, son las preocupaciones señaladas por estas mujeres.

Otra secuela del tratamiento para el cáncer cérvico uterino es la alteración en la percepción de la imagen corporal. Esta alteración se debe a cicatrices producto del tratamiento lo que trae como consecuencia una baja autoestima y una disminución de la confianza en si misma, siendo aún más evidente en las mujeres premenopáusicas $(5,8,10,14,17)$.

Al respecto, y dado los cambios profundos que experimentan las mujeres, existe la necesidad de recibir consejería y apoyo continuo (20). En un estudio realizado por Wenzel y cols (10), se señala que un $69 \%$ de las mujeres recuperadas de $\mathrm{CaCu}$ hubieran deseado consejería al momento del diagnóstico, para resolver preocupaciones relacionadas con efectos de largo plazo del tratamiento, recidiva y aspectos psicosociales.

\section{Dimensión social}

El CaCu al estar relacionado con la promiscuidad sexual trae como consecuencia un estigma social que provoca en la mujer aislamiento por vergüenza, rabia, negación y depresión. El apoyo social de la familia, pareja y amistades constituye la clave para un eficaz afrontamiento y una equilibrada adaptación a la nueva realidad. El no tener una red de apoyo social, puede favorecer el uso de estrategias de afrontamiento poco efectivas tales como el abuso de sustancias y la negación $(10,17)$.

El sistema familiar completo entra en crisis con el diagnóstico de cáncer. Existe un período de reajuste a la nueva situación que requiere de apoyo profesional para afrontar y adaptarse exitosamente. Necesitan estar informados de la enfermedad y clarificar mitos y creencias al respecto (7).

La reinserción de la mujer a las actividades habituales ocurre aproximadamente a los dos años posteriores al tratamiento, ya que antes se ven imposibilitadas de reanudar sus actividades cotidianas, por los efectos secundarios del tratamiento recibido $(9,14)$.

El apoyo social recibido es significativo, en todo momento, pero se vuelve más importante a partir de los seis meses post tratamiento, ya que es ahí cuando la mujer percibe la necesidad de hablar y compartir experiencias de tener cáncer, del tratamiento y de los efectos secundarios de éste, dado que se produce una estabilidad en las alteraciones físicas. También necesitan interactuar con mujeres que han tenido tratamiento similar, para poder hablar con alguien que las pueda entender, escuchar y poder recibir apoyo y reconocimiento de su mejoría y progreso $(5,15,20)$. Un apoyo social indirecto, percibido por las mujeres con $\mathrm{CaCu}$, es el amor por sus hijos y el cuidado continuo de éstos, lo que es considerado la principal motivación para la búsqueda de tratamiento (17).

\section{Dimensión física}

El aspecto físico de la CV de las mujeres con $\mathrm{CaCu}$ es el más valorado según la medicina actual (14). Las alteraciones físicas están sujetas principalmente a la modalidad de tratamiento utilizada. Las pacientes sometidas a radioterapia son las que presentan mayores alteraciones, entre las cuales se encuentran diarrea, poliaquiuria, disuria, secreción vaginal abundante, irritación vaginal, irritación en piel irradiada, cansancio y debilidad. Estos síntomas en el período agudo alteran principalmente el bienestar físico. Tres a cuatro años tras la radioterapia, persisten síntomas como diarrea y fatiga crónica, las que tienen un gran impacto en el deterioro de la función social $(21,22)$. En un estudio realizado por Maduro y cols (15), sobre los efectos secundarios agudos y crónicos presentados por mujeres con cáncer cervical tratadas con radioterapia, se observaron: alteraciones urinarias 27\% (cistitis, fístulas y ulceraciones), dérmicas $20 \%$ (edema, eritema, fibrosis y ulceraciones) y alteraciones genitales en un $12 \%$ de las pacientes (vaginitis, sequedad vaginal, estrechez vaginal, acortamiento vaginal, dispareunia, necrosis cervical, etc.). Los efectos tardíos presentados por la radioterapia fueron gastrointestinales y genitourinarios, los que incluso provocan alteración de la CV general hasta veinte años post tratamiento.

La quimioterapia es la modalidad de tratamiento que presenta alteraciones a corto plazo en todas las dimensiones de la calidad de vida, debido a la sintomatología aguda que ésta provoca. Los efectos secundarios que presentan las mujeres sometidas a éste tratamiento dependen de la terapia utilizada. Durante el período de administración de la quimioterapia existe una gran intensidad de síntomas, los que deterioran la CV general, especialmente el ámbito físico y social, debido al malestar que estos provocan (15).

Las mujeres con $\mathrm{CaCu}$ que son sometidas a mayor número de tratamientos y procedimientos 
médicos presentan peores índices de CV (8). Esto se debe a que presentan mayor número de efectos secundarios de los distintos tratamientos que van deteriorando su bienestar general.

Si se analiza la CV en general, existen factores predictores que la obstaculizan como: menor edad de la paciente, estadio FIGO más avanzado, modalidad de tratamiento (específicamente la radioterapia), mayor número de procedimientos y tratamientos recibidos, menor apoyo social, mayor preocupación relacionada con temas reproductivos, estrategias ineficaces de afrontamiento, salud mental deteriorada y vida espiritual empobrecida o no existente. Los factores favorecedores de la CV son: mayor edad de la paciente, estadio de desarrollo temprano según clasificación FIGO, amplia red de apoyo social, buena comunicación con la pareja especialmente relacionado con temas sexuales, paridad cumplida, estrategias adecuadas de afrontamiento, buen estado de salud mental y vida espiritual activa $(6,7,8,10,14,22)$

\section{CONCLUSIONES}

La CV es un factor importante de valorar en las mujeres con $\mathrm{CaCu}$, ya que esta enfermedad provoca alteraciones en diversos aspectos de la vida. La valoración de la mujer debe ser integral, considerando aspectos físicos, pero también otros aspectos que puedan verse alterados, como son el sexual, social y psicológico.

Es tarea del profesional de la salud detectar alteraciones, que muchas veces son consideradas secundarias por el hecho de centrar la atención sólo en el ámbito físico. Una mirada biopsicosocial de las necesidades de la mujer, permite intervenir de forma oportuna y entregar una atención integral y de calidad a estas usuarias.

En la actualidad, al no existir un instrumento específico para medir CV en mujeres con $\mathrm{CaCu}$, resulta igual de satisfactorio aplicar un cuestionario genérico de la $\mathrm{CV}$, sin embargo, se hace necesario continuar con estudios sobre este tema para así poder llegar a formular un instrumento especifico en este grupo de mujeres.

Es importante identificar factores de riesgos que pueden llegar a deteriorar la CV en mujeres con $\mathrm{CaCu}$, para así poder trabajar en conjunto con un equipo multidisciplinario y reducir al máximo las secuelas sexuales, psicológicas, sociales y físicas que puede dejar esta enfermedad (10). Existen factores predictores que pueden deteriorar la $\mathrm{CV}$ en mujeres con $\mathrm{CaCu}$ y que deben ser utilizados por los profesionales de la salud para identificar a las mujeres con $\mathrm{CaCu}$ que tienen mayor posibilidad de sufrir alteraciones en su CV, y así entregar una atención preventiva oportuna y eficaz.

Los profesionales de salud deben informar a las mujeres con $\mathrm{CaCu}, \mathrm{y}$ a sus familiares, en forma clara y precisa, acerca de las alteraciones que pueden padecer producto de la enfermedad y su tratamiento. Según el estudio realizado por Wenzel y cols (10), mujeres informadas de manera realista sobre su condición, afrontan de una mejor manera las secuelas o limitaciones de la enfermedad a largo plazo. Es importante dar información sobre la evolución de la usuaria, lo que favorecerá un mejor afrontamiento por parte de las mujeres. El entregar información verídica, permitirá crear una relación entre la usuaria y el equipo médico basada en la confianza y en la verdad, aspectos claves para lograr tranquilidad en la usuaria.

El CaCu provoca una crisis existencial tanto en la mujer que lo padece como en su familia. Se destaca que, en las mujeres con $\mathrm{CaCu}$, el recibir consejerías grupales desde el momento del diagnóstico, favorece el buen afrontamiento de la enfermedad. Es importante que en estas consejerías se compartan las experiencias con otras mujeres que han vivido su misma situación y que están en distintos períodos del proceso de enfermedad $(5,15,20,22)$.

Los profesionales del área, tenemos un rol clave en la promoción de una calidad de vida satisfactoria en las mujeres con $\mathrm{CaCu}$, en la prevención de alteraciones en la $\mathrm{CV}$ y en la recuperación de la CV deteriorada por las secuelas de esta enfermedad. Se requiere un rol activo para enfrentar esta problemática, para entregar un cuidado realmente de calidad brindándoles la ayuda necesaria para sobrellevar, y en lo posible mejorar, las múltiples alteraciones que sufren con esta patología (23).

\section{BIBLIOGRAFÍA}

1. MINSAL. Ministerio de Salud de Chile (2003). Recuperado el 27 de septiembre de 2005: http:// deis.minsal.cl/deis/ev/mortalidad_adulta/index.asp

2. Waggoner SE. Cervical cancer. Lancet 2003; 361: 2217-25.

3. MINSAL. Garantías Explícitas en Salud: Guía Clínica Cáncer Cérvicouterino. (2005). Recuperado el 28 de septiembre de 2005: http://www.minsal.cl/ici/ guiasclinicas/CancerCervicouterino.pdf

4. Cantor SB, Fahs MC, Mandelblatt JS, Myers ER, Sanders GD. Decision science and cervical cancer. Cancer Suppl 2003; 98(9): 2003-8. 
5. Juraskova I, Butow P, Robertson R, Sharpe L, McLeod C, Hacker N. Post-treatment sexual adjustment following cervical and endometrial cancer: a qualitative insight. Psychooncology 2003; 12: 26779.

6. Capelli G, De Vicenzo RI, Addamo A, Bartolozzi F, Braggio N, Scambia G. Which dimensions of health related quality of life are altered in patients attending the different gynecologic oncology health care settings? Cancer 2002; 95(12): 2500-7.

7. Eisemann M, Lalos A. Psychosocial determinants of well-being in gynecologic cancer patients. Cancer Nurs 1999; 22(4): 303-06.

8. Hawighorst-Knapstein S, Fusshoeller C, Franz C, Trautmann K, Schmidt M, Pilch $\mathrm{H}$, et al. The impact of treatment for genital cancer on quality of life and body image-results of a prospective longitudinal 10year study. Gynecol Oncol 2004; 94: 398-403.

9. Klee M, Thranov I, Machin D. Life after radiotherapy: the psychological and social effects experienced by women treated for advanced stages of cervical cancer. Gynecol Oncol 2000; 76: 5-13.

10. Wenzel L, DeAlba I, Habbal R, Kluhsman BC, Fairclough D, Krebs LU, et al. Quality of life in longterm cervical cancer survivors. Gynecol Oncol 2005; 97: 310-7.

11. Schwartzmann L. Calidad de vida relacionado con la salud: aspectos conceptuales. Cienc Enferm 2003; 9(2): 9-21.

12. Schover LR. Quality counts: the value of women's perceived quality of life after cervical cancer. Gynecol Oncol 2000; 76: 3-4.

13. WHO. The World Organization quality of life instruments (sf). Recuperado el 27 de Septiembre de 2005: http://www. who.int/evidence/assessmentinstruments/qol/ql1.htm

14. De Groot J, Mah K, Fyles A, Winton S, Greenwood $S$, Depetrillos A, et al. The psychosocial impact of cervical cancer among affected women and their partners. Int J Gynecol Cancer 2005; 15: 918-25.

15. Maduro JH, Pras E, Willemse PHB, de Vries EGE. Acute and long term toxicity following radiotherapy alone or in combination with chemotherapy for locally advanced cervical cancer. Cancer Treatment Reviews 2003; 29: 471-88.

16. Jensen PT, Groenvold M, Klee MC, Tharanov I, Petersen MA, Machin D, Stat C. Longitudinal study of sexual function and vaginal changes after radiotherapy for cervical cancer. Int J Radiat Oncol Biol Phys 2003; 56(4): 937-49.

17. Ashing-Giwa KT, Kagawa-Singer M, Padilla GV, Tejero JS, Hsiao E, Chhabra R, et al. The impact of cervical cancer and dysplasia: a qualitative, multiethnic study. Psychooncology 2004; 13: 709-28.

18. Li C, Samsioe G, losif C. Quality of life in long-term survivors of cervical cancer. Europ Menopause $\mathrm{J}$ 1999; 32: 95-102.

19. Greimel E, Thiel I, Peintinger F, Cegnar I, Pongratz E. Prospective assessment of quality of life of female cancer patients. Gynecol Oncol 2002; 85: 140-7.

20. Basen-Engquist K, Paskett ED, Buzaglo J, Miller SM, Schover L, Wenzel LB, et al. Cervical Cancer. Behavioral factors related to screening, diagnosis and survivors' quality of life. Cancer Suppl 2003; 98(9): 2009-13.

21. Bye A, Tropé C, Loge JH, Hjermstad M, Kaasa S. Health related quality of life and ocurrence of intestinal side effects after pelvis radioterapy. Acta Oncol 2000; 39(2): 173-80.

22. Klee M, Thranov I, Machin D. The patients' perspective on physical symptoms after radiotherapy for cervical cancer. Gynecol Oncol 2000;76:14-23.

23. Hilton LW, Jennings-Dozier K, Bradley PK, LockwoodRayermann S, De Jesus Y, Stephens DL, et al. The role of nursing in cervical cancer prevention and treatment. Cancer Suppl 2003; 98(9): 2070-4. 